Supplementary Material for:

\title{
Heme protein oxygen affinity regulation exerted by proximal effects
}

Luciana Capece, Marcelo A. Marti, Alejandro Crespo, Fabio Doctorovich and Darío A. Estrin*

Departamento de Química Inorgánica, Analítica y Química Física / INQUIMAE-CONICET, Facultad de Ciencias Exactas y Naturales, Universidad de Buenos Aires, Ciudad Universitaria, Pabellón 2, Buenos Aires, C1428EHA, Argentina.

*To whom correspondence should be addressed. E-mail: dario@qi.fcen.uba.ar, Fax:(5411) 45763341

\section{Computational Methodology}

The quantum subsystem in our scheme is treated at the density functional theory (DFT) level using the efficient SIESTA (Spanish Iniciative for the Electronic Structure of Thousands of Atoms) implementation. ${ }^{1}$ The use of standard norm-conserving pseudopotentials ${ }^{2}$ avoids the computation of core electrons, smoothing at the same time the valence charge density. We have used the TroullierMartins scheme with pseudopotential radii for Fe orbitals of 2.3 bohrs, and for all other elements orbitals of 2.0 bohrs. In our study, the nonlinear partial-core correction ${ }^{3}$ is applied to the iron atom.

Basis functions consisting of localized (numerical) pseudoatomic orbitals, are projected on a real space grid to compute the Hartree potential and exchange correlation potentials matrix elements. For all atoms, basis sets of double plus polarization quality were employed, with a pseudoatomic orbital energy shift of $25 \mathrm{meV}$ and a grid cutoff of $150 \mathrm{Ry} .{ }^{1,4}$ The supercell geometry was chosen large enough for the 
interaction between systems in neighboring cells to be negligible. Calculations were performed using the generalized gradient approximation functional proposed by Perdew, Burke and Ernzerhof..$^{5}$ This combination of pseudopotentials, density functional, basis sets, and grid parameters has been already validated for heme models. ${ }^{4}$ Geometry optimizations were performed using a conjugate gradient algorithm.

\section{$\mathrm{O}_{2}$ binding energy}

The $\mathrm{O}_{2}$ binding energy ( $\Delta \mathrm{E}_{\mathrm{O} 2}$ ) was calculated using the following equation:

$$
\Delta \mathrm{E}_{\mathrm{O} 2}=\mathrm{E}_{\mathrm{Heme}-\mathrm{O} 2}-\mathrm{E}_{\mathrm{Heme}}-\mathrm{E}_{\mathrm{O} 2}
$$

where $\mathrm{E}_{\mathrm{Heme}-\mathrm{O} 2}$ is the energy of the oxy heme complex, $\mathrm{E}_{\mathrm{Heme}}$ is the energy of the free heme group and $\mathrm{E}_{\mathrm{O} 2}$ is the energy of the isolated oxygen molecule, where $\mathrm{E}_{\mathrm{Heme}-\mathrm{O} 2}$ is the energy of the oxy heme complex (treated as a low spin singlet state), $\mathrm{E}_{\mathrm{Heme}}$ is the energy of the free (de-oxy) heme group (treated as a high spin quintuplet state) and $\mathrm{E}_{\mathrm{O} 2}$ is the energy of the isolated oxygen molecule. These values were calculated in the following way:

- Model systems presented in Table 1 (contribution from the charge relay mechanism):

QM optimizations of the oxy and de-oxy complexes were performed for each model. The heavy atoms of the extra groups corresponding to each model were fixed during the simulations.

In addition, single point calculations were performed on the oxygenated and deoxygenated optimized structures for the three models, adding diffuse functions for nitrogen, oxygen and hydrogen atoms. For this purpose, we used the following basis specifications, in which the numbers (bold) correspond to the cutoffs of the additional functions:

\%block PAO.Basis

C 2 \# Species label, number of 1-shells 


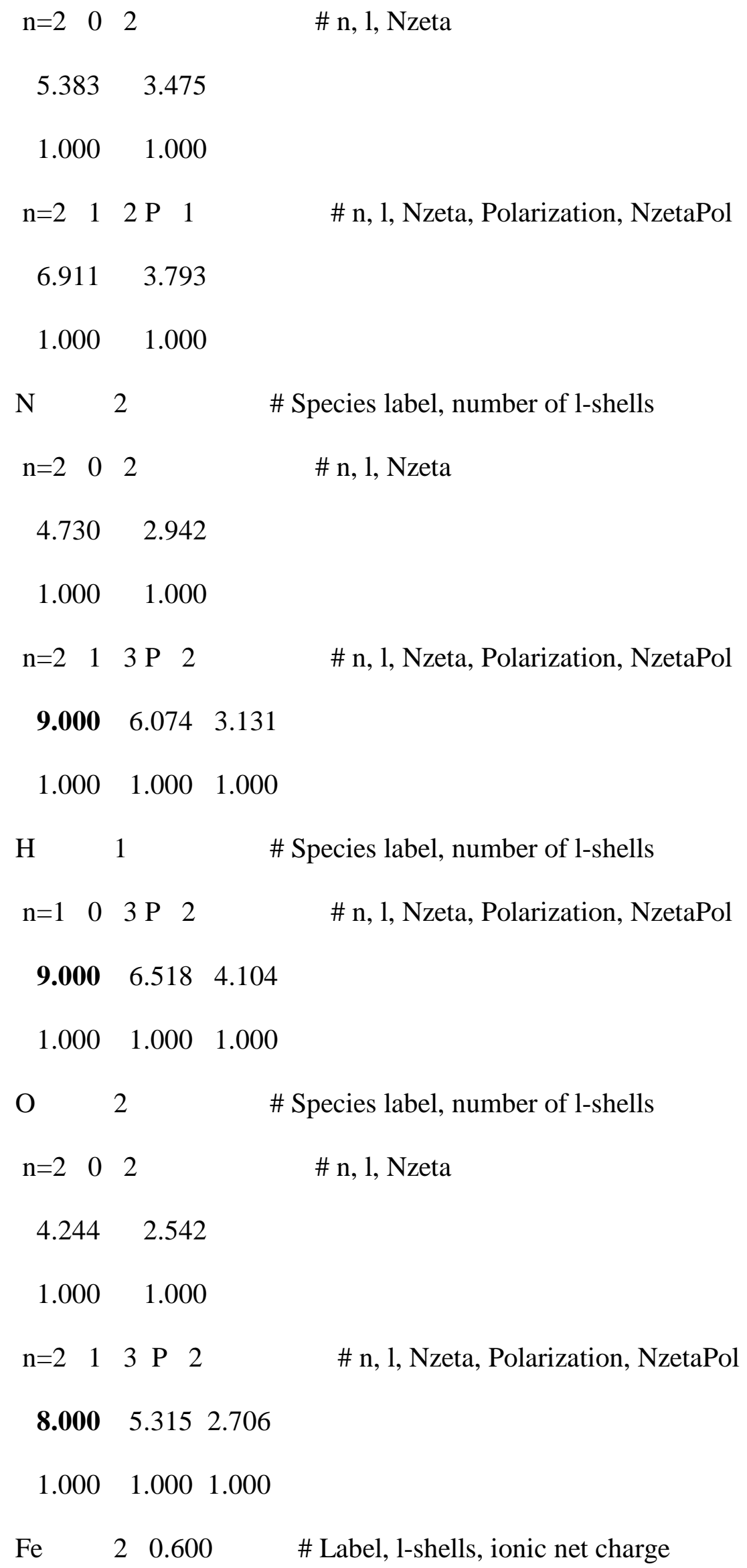




$\begin{array}{lllll}\mathrm{n}=4 & 0 & 2 \mathrm{P} & 1 & \text { \# n, l, Nzeta, } \\ 7.705 & 5.708 & \\ 1.000 & 1.000 & \\ \mathrm{n}=3 & 2 & 2 & \\ 4.792 & 2.153 \\ 1.000 & 1.000\end{array}$

- Model systems presented in Table 2 (contribution from the proximal histidine conformation).

QM-MM restricted optimizations were performed for the oxy and de-oxy complexes in staggered and eclipsed conformation. The heme group, the $\mathrm{O}_{2}$ and the imidazole were treated quantum-mechanically, while the acetone molecule was treated classically. The dihedral angle was restrained to the desired value during the simulations, adding the following term to the potential energy:

$$
V_{R}=\frac{1}{2} k\left(\xi-\xi_{O}\right)^{2}
$$

with $\mathrm{k}=200 \mathrm{kcal} /\left(\mathrm{mol} . \theta^{2}\right)$ and $\xi_{\mathrm{o}}=0$ for the eclipsed conformation and $\xi_{\mathrm{o}}=\pi / 4$ for the staggered conformation.

- Model system presented in Figure 4 (effect of the Fe-His distance)

QM restricted optimizations were performed for the oxy and de-oxy complexes. In each calculation, the distance between the $\mathrm{N} \varepsilon$ of the proximal imidazole and the center of the four nitrogen atoms of the porphyrine was restrained to the desired value for each simulation, with $\mathrm{k}=200 \mathrm{kcal} /\left(\mathrm{mol} . \AA^{2}\right)$ and $\xi_{\mathrm{o}}$ from 1.95 to $2.25 \AA$.

The $\Delta \mathrm{E}_{\text {comp }}$ values were calculated as the difference between the $\mathrm{O}_{2}$ binding energy of the complex in the restrained conformation minus the $\mathrm{O}_{2}$ binding energy of the complex in the equilibrium: 
$\Delta \mathrm{E}_{\text {comp }}=\Delta \mathrm{E}_{\mathrm{O} 2 \text { (restrained) }}-\Delta \mathrm{E}_{\mathrm{O} 2 \text { (equilibrium) }}$

- QM-MM oxygen affinity calculations on myoglobin and leghemoglobin w.t and mutants.

The oxy complexes of each protein were obtained by performing full QM-MM optimizations. In order to obtain the vertical $\mathrm{O}_{2}$ binding energy, we calculated the deoxygenated proteins frozen in the optimized oxygenated conformation. The QM subsystems were treated at the DFT level as described above, whereas the classical subsystems were treated using the Amber99 force field parametrization. . We have selected the iron porphyrinate plus the axial ligands as the quantum subsystem. The rest of the protein unit mentioned above and the water molecules were treated classically. The frontier between the QM and MM portions of the system has been treated by the scaled position link atom method. ${ }^{7}$ Link $^{2}$ atoms have been used in the proximal histidine to separate the QM treated imidazole ring from the backbone of the aminoacids and to separate the core of the porphyrin from its side chains.

The Lennard-Jones parameters used for the QM atoms in the QM-MM interaction terms ${ }^{8}$ might adopt larger values than the Lennard-Jones parameters of the MM atoms. ${ }^{9}$ In order to analyze the influence of this issue we recalculated the $\mathrm{O}_{2}$ binding energy for $\mathrm{Mb}$ w.t. and HisE7xGly mutant, using $10 \%$ increased $\sigma \mathrm{LJ}$ parameters for the oxygen atoms. The difference in binding energy between the w.t. and the distal mutant resulted $8 \mathrm{kcal} / \mathrm{mol}$, which is very close to the value reported in the manuscript $(7.1 \mathrm{kcal} / \mathrm{mol})$. It is worth mentioning that the main geometrical parameters have not changed significantly in these new calculations. The PDB structures of the proteins calculated with the both LJ sets are included as supplementary material files.

- $\quad$ wtmb.pdb (w.t. Mb calculated with the original Lennard-Jones parameters)

- $\quad$ hise7xglymb.pdb (HisE7xGly mutant calculated with the original Lennard-Jones parameters)

- $\quad$ wtmbinclj.pdb (w.t. Mb calculated with the increased $\sigma$ Lennard-Jones parameters)

- hise7xglymbinclj.pdb (HisE7xGly mutant calculated with the increased $\sigma$ Lennard-Jones parameters) 
(1) Soler, J. M.; Artacho, E.; Gale, J.; García A.; Junquera, J. Ordejón, P.; Sánchez-Portal D. J. Phys: Cond. Matt. 2002, 14, 2745-2779.

(2) Troullier, N., Martins, J. L. Phys. Rev. B 1991, 43, 1993-2006.

(3) Louie, S. G., Froyen, S., Cohen, M. L. Phys. Rev. B 1982, 26, 1738-1742.

(4) Martí, M.A., Scherlis, D.A., Doctorovich, F.A., Ordejón, P., Estrin, D.A. J. Biol. Inorg. Chem. 2003, 6, 595-600.

(5) Perdew, J. P., Burke, K.; Ernzerhof, M. Phys. Rev. Lett. 1996, 77, 3865-3868.

(6) Wang, J.; Cieplak, P.; Kollman, P.A. J. Comput. Chem. 2000, 21, 1049-1074.

(7) a) Eichinger, M.; Tavan, P.; Hutter, J.;Parrinello, M. J. Chem. Phys. 1999, 110, 10452-10467. b) Rovira, C.; Schulze, B.; Eichinger, M.; Evanseck, J.D.; Parrinello, M. Biophys J. 2001, 81, 435-445.

(8) Crespo, A.; Scherlis, D.A.; Martí, M.A.; Ordejón, P.; Roitberg, A.E.; Estrin, D.A. J. Phys. Chem. B 2003, $107,13728-13736$.

(9) Murphy, R.B.; Philipp, D.M.; Friesner, R.A. J. Comput. Chem 2000, 21, 1442-1457. 